\title{
Effect of Cage Therapy using Advanced Spider Suit Compared to Traditional Physical Therapy on Gross Motor Function in Children with Cerebral Palsy - An Indian Experience
}

\author{
Kanu Kaushik $^{1}$ and Kishore Kumar ${ }^{1,2^{*}}$ \\ ${ }^{1}$ Cloudnine Hospital, Jayanagar, Bangalore, India \\ ${ }^{2}$ Notre Dame University, Perth, Australia
}

${ }^{*}$ Corresponding author: Kishore Kumar, Consultant Neonatologist and Pediatrician, Cloudnine Hospital, 1533, 3rd Block Jayanagar, 9th Main, Bangalore - 560011, India, Tel: + 91804020 2222; Fax: + 91804020 2233; E-mail: drkishore@cloudninecare.com

Rec date: Jan 19, 2016; Acc date: Feb 12, 2016; Pub date: Feb 18, 2016

Copyright: ( 2016 Kaushik K, et al. This is an open-access article distributed under the terms of the Creative Commons Attribution License, which permits unrestricted use, distribution, and reproduction in any medium, provided the original author and source are credited.

\begin{abstract}
Objectives: Cerebral Palsy $(\mathrm{CP})$ is a condition where non progressive disorders of posture caused by abnormal development of, or damage to, motor control centers of brain resulting in abnormal movements. Improving gross motor function in children with cerebral palsy has been a major aim of physical therapist. The purpose of this study is to find out the Effect of Cage Therapy using Advanced Spider Suit Compared to Traditional Physical Therapy on Gross Motor Function in Children with Cerebral Palsy.
\end{abstract}

Materials and Methods: A comparative study was conducted to find out the effect of Cage Therapy using Advanced Spider Suit Compared to Traditional Physical Therapy on Gross Motor Function in Children with Cerebral Palsy.

10 children with cerebral palsy were selected for this intervention. Age group between 4-8 years With GMFCS level III-IV were selected for this study.

In 10 children we have 2 Hypotonic, 2 hemiplegic and 6 Spastic diplegic kids. These subjects were equally distributed in each group.

Intervention: Therapy lasted for 8 weeks (5 days a week and $2 \mathrm{hrs} / \mathrm{day}$ ).

Group A - 5 subjects were subjected to Cage therapy using advanced spider suit.

Group B -5 subjects were subjected to traditional physical therapy.

Gross Motor Function Measure was used to assess gross motor abilities of the subjects before and after intervention.

Results: The result showed significant improvement with Cage therapy using advanced spider suit therapy than traditional physical therapy on Gross Motor Function Measure.

Conclusions: The Cage Therapy using Advanced Spider Suit is more effective and beneficial therapy than Traditional Physical Therapy in improving Gross Motor Function in Children with Cerebral Palsy.

\section{Keywords Cage Therapy; Spider Suit; Cerebral Palsy; GMFM}

\section{Introduction}

Cerebral palsy is a condition where non progressive disorders of posture caused by abnormal development of, or damage to, motor control centers of brain resulting in abnormal movements - attributed to non-progressive disturbances that occur in the developing brain. The motor disorders of cerebral palsy are often accompanied by disturbances of sensation, cognition, communication, perception and behavior and by seizure disorder [1]. Around 8000-10000 babies and infants are diagnosed annually with cerebral palsy. Cerebral palsy is the second most common neurological impairment in childhood. The incidence of cerebral palsy is estimated to be around 2-2.5 per 1000 live births [2].

Over the past fifty years there is emphasis on prevention of $\mathrm{CP}$, though research into affected children to promote gross motor development for children with Cerebral Palsy (CP) has focused on rehabilitation method, training method, or treatment method. However many of these studies have been inconclusive secondary to methodological bias or limitation such as small samples, inappropriate outcome measures, improper study design, or lack of standardization of experimental procedures [3]. 
Citation: Kaushik K, Kumar K (2016) Effect of Cage Therapy using Advanced Spider Suit Compared to Traditional Physical Therapy on Gross Motor Function in Children with Cerebral Palsy - An Indian Experience. Int J Neurorehabilitation 3: 1000193. doi: $10.4172 / 2376-0281.1000193$

Page 2 of 5

Gross Motor Function is a major debilitating factor for the performance of activities of daily living in children with cerebral palsy. Recent evidence suggests that children with $\mathrm{CP}$ may improve gross motor function if provided opportunities to practice in right way.

One treatment approach that is becoming popular is Cage Therapy along with Advanced Spider Suit.

The Cage Therapy Unit is a unique and dynamic device consisting of a system of pulleys, straps, and splints utilized to perform a variety of exercise. This system improves strength, passive and active range of motion, and muscle flexibility. With the use of this system, the therapist can isolate any muscle group and target. In this situation the muscle tone (usually increased) does not influence the movements. This allows muscle groups to counteract the spastic muscles. The end effect is functional gains. The quality of gait, balance, and coordination of movements increases rapidly.

The key elements of Cage Therapy Unit are:

The "Monkey Cage" is a tri-dimensional rigid metal cage where metal pulleys are arranged to stretch and strengthen muscles groups.

The "Spider Cage", therapist uses leather belt in which bungee cords are connected. In this way, the patient is supported and can safely learn weight shift, jump, kneel, half-kneel and step up and over objects. The "spider cage" is an effective tool for implementing neuro-development treatment (NDT), one of the most wide-spread and clinically accepted methods for "re (programming)" the central nervous and neuromuscular systems and "teaching" the brain more adequate motor skills.

Advanced Spider Suit is a soft dynamic orthotic which consists of a cap, vest, shorts, knee pads and special adapted shoes that are interconnected with elastic bands. The basic concept of the Advanced Spider Suit is to create a supporting unit to align the body as close to normal as possible re-establishing correct postural alignment and weight bearing which is fundamental in normalizing muscle tone, sensory and vestibular function. The elastic bands are adjustable, which means that we can load the body axially within 15 to $40 \mathrm{~kg}$.

The Advanced Spider Suit is the most modern type of suit available today.

Traditional Physical Therapy Program is designed of specific sets of exercises to work towards 3 important goals:

a) To maintain or improve muscle property

b) To avoid contractures and to improve the child's motor development

c) Activities and muscle re-education exercises to improve flexibility, strength, balance \& co-ordination.

Strengthening muscles and improving function, contributes to gains in range of motion, both active and passive, and improved muscle flexibility and tone, promoting good benefits $[7,8]$ as for the cerebral palsy population. According to $\mathrm{Wu}$ et al. [9], passive stretching combined with engaging in active movement training with $\mathrm{CP}$ children demonstrated improvements in joint biomechanical properties, motor control performance, and functional capability in balance and mobility. Children's physical development and movement is linked closely to other aspects of their daily learning. It is influenced by their growing confidence and enjoyment of physical play, by their increasing ability to control their own bodies through movement and by their physical well-being and strength [10].

\section{Materials and Methods}

\section{Study design}

A comparative study was conducted to find out the effect of Cage Therapy using Advanced Spider Suit Compared to Traditional Physical Therapy on Gross Motor Function in Children with Cerebral Palsy.

\section{Sample}

10 subjects were selected after giving due consideration to inclusion and exclusion criteria. In 10 children we have 2 Hypotonic, 2 hemiplegic and 6 Spastic diplegic kids.

\section{Sampling method}

Random sampling technique was used to select the samples.

\section{Inclusion criteria}

Diagnosis of Cerebral palsy

Both gender with children aged 4 to 8 years

Gross Motor Function Classification System level III-V.

\section{Exclusion criteria}

Subluxation or dislocation of hip exceeding 33\%

Scoliosis exceeding 25 degrees

Osteoporosis

High blood Pressure

Complicated systemic disorders

Uncontrollable seizures

Usage of Botulinum toxin A injection within 3 months of prior study.

\section{Outcome Measures}

\section{Gross motor function measure (GMFM)}

The Gross Motor Function Measure (GMFM) is a clinical tool designed to evaluate change in gross motor function in children with cerebral palsy. There are two versions of the GMFM - the original 88item measure (GMFM-88) and the more recent 66-item GMFM (GMFM-66). Items on the GMFM-88 span the spectrum from activities in lying and rolling up to walking, running and jumping skills. The GMFM-66 is comprised of a subset of the 88 items identified (through Rasch analysis) as contributing to the measure of gross motor function in children with cerebral palsy. The GMFM-66 provides detailed information on the level of difficulty of each item thereby providing much more information to assist with realistic goal setting.

This is a single test retest case report using the Gross Motor Function Measure-88 (GMFM-88). A baseline measurement was taken to provide a basis for comparison with the new intervention. The GMFM-88 baseline measurement was scored two times: once without AFO's and assistive devices and a second time with AFO's and assistive devices. The GMFM is a criterion-referenced measure based on the 
Citation: Kaushik K, Kumar K (2016) Effect of Cage Therapy using Advanced Spider Suit Compared to Traditional Physical Therapy on Gross Motor Function in Children with Cerebral Palsy - An Indian Experience. Int J Neurorehabilitation 3: 1000193. doi: $10.4172 / 2376-0281.1000193$

Page 3 of 5

concepts of abilities and limitations in gross motor function and is analogous to the staging and grading systems.

\section{Procedure}

Ten subjects aged between 4-8 years with cerebral palsy were selected based on inclusion and exclusion criteria and divided into two groups namely Group A and Group B. All these subjects were assessed using a general assessment pro forma that recorded Gross Motor Function Measure.

\section{Group A}

The subject received two hours/ day for five days in a week for two months of Cage therapy with Advanced Spider suit which includes:

a) General warm up and mild stretches followed by Monkey Cage therapy - Basically to improve strength by isolating muscle group without compensating with other muscle group (weights and pulley system)

b) Spider Cage therapy to improve Co-ordination and Balancing and assisted movements like sit to stand, quadruped, squats, and jumping.

The therapist guides the child through exercises to strengthen muscles and allow the patient to experience movements.

\section{Group B}

The subject received Traditional Physical therapy for two hours/ day for five days in a week for two months which includes:

a) General body warm up exercises

b) Passive and active assisted range of motion exercises for all the joints.

c) Muscle Stretches and flexibility exercises

d) Strengthening exercises with weights and resistance band.

e) Balancing and co-ordination exercises

\section{Results}

The aim of the study was to find out the effectiveness of Cage Therapy using Advanced Spider Suit Compared to Traditional Physical Therapy on Gross Motor Function in Children with Cerebral Palsy.

Histogram 1-3 shows Group A and B Motor Function Measure Pre and Post Test Result.

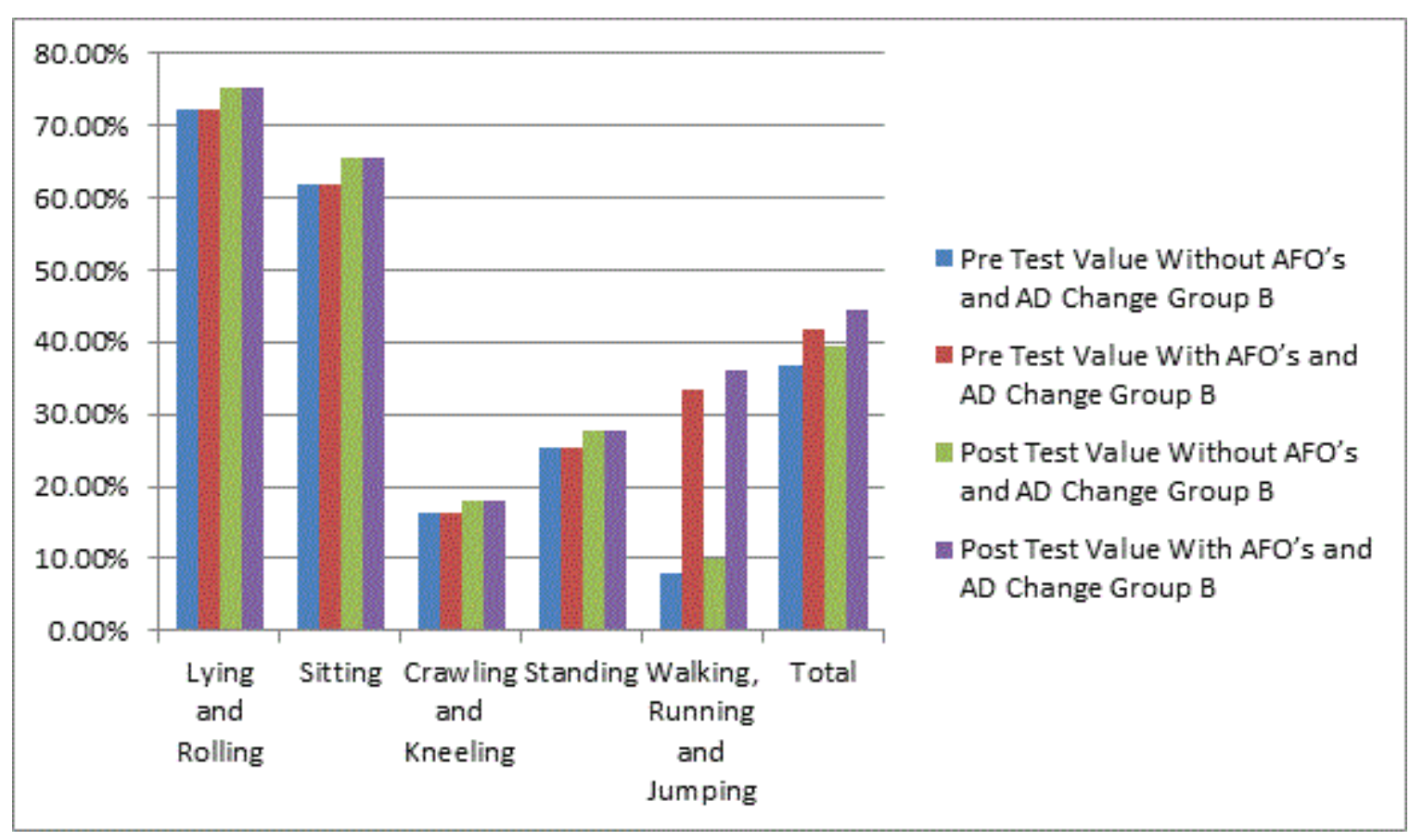

Histogram 1: Group A gross motor function measure pre and post test result. 
Citation: Kaushik K, Kumar K (2016) Effect of Cage Therapy using Advanced Spider Suit Compared to Traditional Physical Therapy on Gross Motor Function in Children with Cerebral Palsy - An Indian Experience. Int J Neurorehabilitation 3: 1000193. doi: $10.4172 / 2376-0281.1000193$

Page 4 of 5

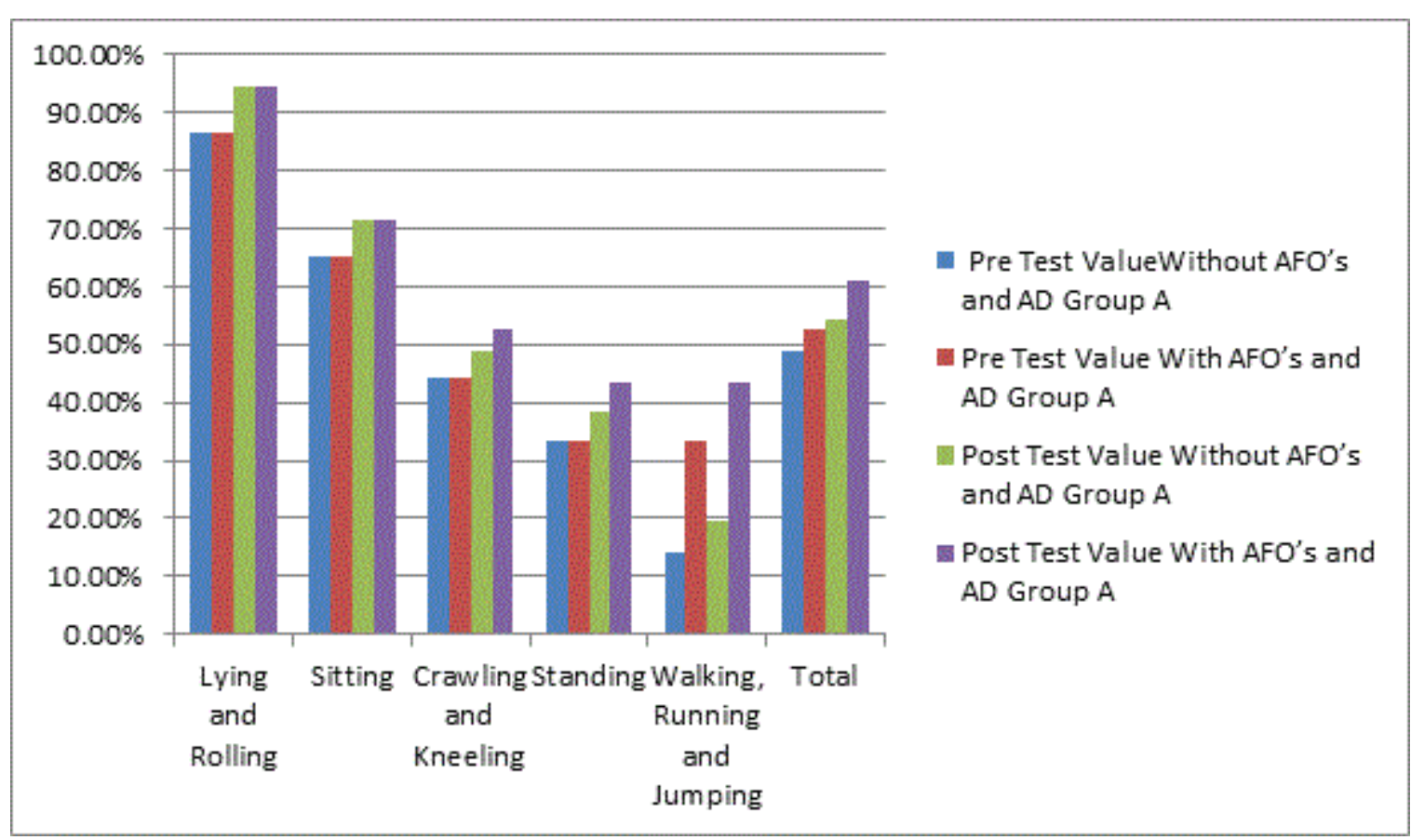

Histogram 2: Group B gross motor function measure pre and post test result.

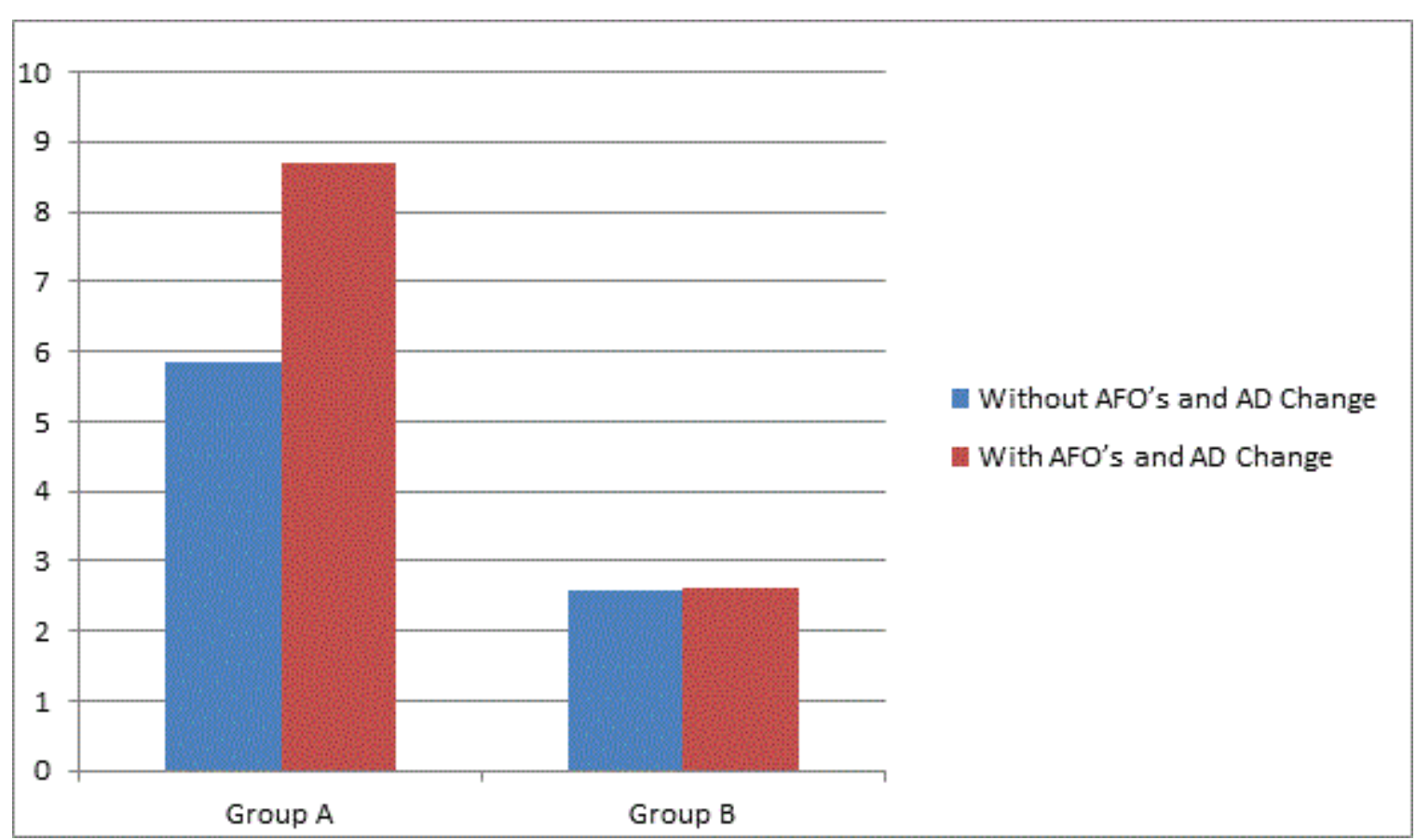

Histogram 3: Post-test comparison between Group A and B.

Table 1 displays the GMFM values of Post-test therapy program. The result showed significant difference in improvement of GMFM values in GROUP A (Without AFO's and AD Change $=+5.84$, With
AFO's and AD Change $=+8.71$ ) which is greater than the Group $\mathrm{B}$ (Without AFO's and AD Change $=+2.58$, With AFO's and AD Change $=+2.63)$. 
Citation: Kaushik K, Kumar K (2016) Effect of Cage Therapy using Advanced Spider Suit Compared to Traditional Physical Therapy on Gross Motor Function in Children with Cerebral Palsy - An Indian Experience. Int J Neurorehabilitation 3: 1000193. doi: $10.4172 / 2376-0281.1000193$

Page 5 of 5

\begin{tabular}{|l|l|l|l|}
\hline Group A & & Group B & \multirow{2}{*}{ With AFO's and AD Change } \\
\hline Without AFO's and AD Change & With AFO's and AD Change & Without AFO's and AD Change & +2.63 \\
\hline+5.84 & +8.71 & +2.58 & + \\
\hline
\end{tabular}

Table 1: Post-test comparison

\section{Discussion}

Present study was done to find out the effectiveness of Cage Therapy using Advanced Spider Suit Compared to Traditional Physical Therapy on Gross Motor Function in Children with Cerebral Palsy (Figure 1).

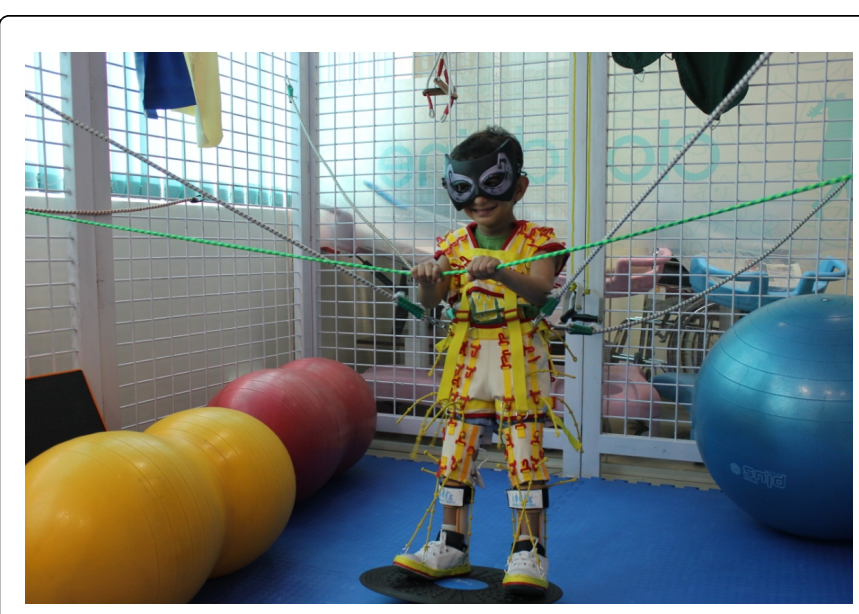

Figure 1: Subject with Spider suit performing exercises in Cage Therapy Unit.

This Advanced therapy had been proven to be effective in improving Gross Motor Function in children with cerebral palsy in this study with limited number of children. The reason behind this improvement can be the number of sessions of therapy (2hours/day and 5 days a week for 2 months), Benefit of Advanced Spider Suit which provides external stabilization to the trunk and therefore allows more fluent and coordinated movement for both upper and lower extremities with combination of Spider Cage Therapy and Monkey Cage Therapy. Large multi-centre randomized control trials are essential to confirm our findings. But this provides a promising future with better patient satisfaction for children with CP.

\section{Conclusion}

The Cage Therapy using Advanced Spider Suit is more effective and beneficial therapy than Traditional Physical Therapy in improving Gross Motor Function in Children with Cerebral Palsy.
The Cage Therapy using Advanced Spider Suit is customized to the needs of each cerebral palsy child, with specific functional goals and usually involves an intensive rehabilitation program. It combines the best elements of various techniques and methods. It is important to note that the use of the suit is only one component of an overall approach. Parental involvement is highly encouraged and parents are often part of the treatment program. It needs to be mentioned, however, that a true intensive therapy program is not merely doing the same exercise over and over again, but rather is a structured approach to physical activity with consideration to the person's individual cycles of progressive overload, fatigue and recovery.

\section{References}

1. United Cerebral palsy Research and Educational Foundation (2005) Developmental medicine and Child Neurology 47: 571-576.

2. Rosen MG, Dickinson JC (1992) The incidence of cerebral palsy. Am J Obstet Gynecol 167:417-423.

3. Trahan J, Malouin F (2002) Intermittent Intensive Physiotherapy in Children with Cerebral Palsy: A Pilot Study. 44: 233-239.

4. Nordmark E, Hagglund G, Jarnio GB (1997) Reliability of the Gross Motor Function Measure in Cerebral Palsy. Scand J Rehabil Med 29: 25-28.

5. Russel DJ, Rosenbaun PL, Avery LM (2002) Gross Motor Function Measure (GMFM-66 \& GMFM-88) User's Manual. London, England: Mac Keith Press.

6. Palisano RJ, Hanna SE, Rosenbaum PL (2000) Validation of a Model of Gross Motor Function for Children with Cerebral Palsy. Phys Ther 80: 974-983.

7. Marek SM, Cramer JT, Fincher AL, Massey LL, Dangelmaier SM, et al. (2005) Acute effects of static and proprioceptive neuromuscular facilitation stretching on muscle strength and power output. J Athl Train 40: 94-103.

8. Cramer JT, Housh TJ, Weir JP, Johnson GO, Coburn JW, et al. (2005) The acute effects of static stretching on peak torque, mean power output, electromyography, and mechanomyography. Eur J Appl Physiol 93: 530-539.

9. Wu YN, Hwang M, Ren Y, Gaebler-Spira D, Zhang LQ (2011) Combined passive stretching and active movement rehabilitation of lower-limb impairments in children with cerebral palsy using a portable robot. Neurorehabil Neural Repair 25: 378-385.

10. Scholtes V, Dallmeijer A, Rameckers E, Verschuren O, Tempelaars E, et al. (2008) Lower limb strength training in children with cerebral palsy-a randomized controlled trial protocol for functional strength training based on progressive resistance exercise principles. BMC Pediatr $8: 41$. 